\title{
The Designing and Applications of Expert Computer Systems in the Sciences
}

\author{
Parag Rastogi \\ Assistant Professor \\ Department of Information Technology \\ Bharat Institute of Technology, Meerut(UP) INDIA
}

\begin{abstract}
The credibility of AI rose to new heights in the minds of individuals and critics when many Expert Systems(ES) were successfully planned, developed, and implemented in many challenging areas. As of today, quite a heavy investments is done in this sector. The success of these programs in very selected fields involving high technical expertise has left people to explore new avenues. An expert computer system is a computer program which captures the knowledge of a human expert on a given problem, and applies this knowledge to solve complex problems in a manner similar to the expert. This paper describes important concepts pertaining to the expert computer systems, highlights the major characteristics, structure and design methodology of the expert systems. This paper also reviews the different applications of expert systems in various classes of science.
\end{abstract}

Keywords - expert computer systems, heuristic-knowledge, inference engine, knowledge base, knowledge acquisition.

\section{INTRODUCTION}

One of the goals of AI is to understand the concept of intelligence and develop intelligent computer programs. The most important applied area of AI is the field of expert systems. An Expert System (ES) is a knowledge based system that employs knowledge about its application domains and uses an inferencing (reasoning) procedure to solve problems that would otherwise require human competence or expertise. The power of expert systems stems primarily from the specific knowledge about a narrow domain stored in the expert system's knowledge base.

It is important to understand that the expert systems are assistants to decision makers and not substitutes for them. Expert systems do not have human capabilities. They use a knowledge base of a particular domain and bring that knowledge to bear on the facts of the particular situation at hands. The knowledge base of an expert system also contains heuristic-knowledge_rules of thumb used by human expert who work in the domain.

Expert System technology has captured the interest of professionals in a number of fields in recent years. Systems have been developed in such diverse areas as science, engineering, business and medicine. Almost every professional and computer society currently has a special interest group for expert systems technology. This wide spread interest can be attributed to the ability of the expert system to aid various organizations involving practical real world problems. Currently 1000 companies have expert systems projects under development.

In this paper it is described an organizational structure of a strategic process for developing and designing successful expert systems. There are several topics in hierarchy of designing an expert system. The strategic process is recommended for anyone venturing into technology for expert system from standpoint of training, research, or applications. This paper covers all the fundamental concepts of an expert computer system.

\section{CHARACTERSTICS OF AN EXPERT COMPUTER SYSTEM}

The main characterstics of expert computer system are as follows :

1. They reduce the cost of accessing information as it allows dissemination of information held by one or small group of experts to less expensive people.

2. Knowledge in an expert system can be formalized, tested, and validated.

3. They allow integration from different sources.

4. The response time of an expert system should be adequate.

5. Increase the probability, frequency and consistency of making good decision. 
6. They use symbolic knowledge representation.

7. Help distribute human expertise.

8. Expert systems often reason with meta-knowledge; that is, they reason with knowledge about themselves, and their own knowledge limits and capabilities.

\section{DIFFERENCE BETWEEN EXPERT SYSTEM AND SOFTWARE SYSTEM}

\begin{tabular}{|c|c|}
\hline \multicolumn{1}{|c|}{ Expert System } & Software System \\
\hline $1 . \quad$ They use knowledge base & 1. They use database \\
\hline $2 . \quad$ Lesser data is kept together & 2. Large data is kept separately \\
\hline 3. They use reasoning mechanism & 3. They use algorithms \\
\hline 4. They use inference engine & 4. They use compilers \\
\hline 5. $\quad \begin{array}{l}\text { They are developed by knowledge engineers } \\
\text { e.g. DENDRAL,MYCIN, LITHO, TIMM, } \\
\text { XCON, CLIPS etc. }\end{array}$ & e.g. C, C++, JAVA etc. \\
\hline
\end{tabular}

But we should note that knowledge base is different from a database:

\begin{tabular}{|c|c|c|}
\hline \multicolumn{2}{|c|}{ Knowledge base } & Database \\
\hline $\begin{array}{l}\text { 1. It stores information at a higher level of } \\
\text { abstraction. }\end{array}$ & 1. They are a collection of data representing facts \\
\hline 2. & It operates on a class of objects & 3 It operates on single object \\
\hline 3. & They use power of inferencing & Information needs to be explicitly stated \\
\hline 4 & $\begin{array}{l}\text { Representation is by logic or rules or frames or } \\
\text { scripts or nets. }\end{array}$ & 5. It is maintained for operational purpose only. \\
\hline 5. & It is used for data analysis and planning.
\end{tabular}

\section{NEED OF AN EXPERT COMPUTER SYSTEM AND ITS JUSTIFICATION}

A human expert is perishable but artificial expert system is not. It is permanent. If there is one expert then the problem cannot be solved if that expert is not experienced. So we take group of experts as then the solution is more efficient. One expert computer system can be used at different places at the same time. Expert System are tools of mass production. Emotions also have no affect on expert computer systems.

In nutshell, an ES is like a tutor for someone who is interested in learning. Expert Systems have variety of applications - medicine, engineering, geology, information technology, basic and applied research, training, sales and marketing.

\section{PHASES IN DESIGNING EXPERT SYSTEMS}

Designing an expert system initially requires extracting the relevant knowledge from a human domain expert; e is almost subconscious this knowledge is often based on useful thumb rules, and experience rather than absolute certainties. Usually experts find it difficult to express concretely the knowledge and rules used by them, while solving a problem. This is because their knowledge is almost subconscious or appears so obvious that they do not bother mentioning it. After extracting knowledge from domain experts the next step is to represent this knowledge in the system. Representation of knowledge in a computer system is not straight forward and requires special expertise. A knowledge engineer handles the responsibility of extracting this knowledge and building the expert computer system's knowledge base. This process of gathering knowledge from a domain expert and codifying it according to formalism is known as knowledge engineering. This phase is known as knowledge acquisition, which is a big area of research.

To be more precise, the different independent and overlapping phases involved in designing an expert system may be categorized as follows : 
(i) Identification phase: In this phase the knowledge engineer determines important features of the problem with the help of human domain expert. The parameters that are determined in this phase include the type and scope of the problem, the kind of resources required, and the goal and objective of the expert system.

(ii) Conceptualization phase: In this phase knowledge engineer and domain expert decide the concepts, relations and control mechanism needed to describe the problem-solving method. At this stage, the issue of granularity is also addressed, which refers to the level of details required in the knowledge.

(iii) Formalization phase: This phase involves expressing the key concepts and relations in some framework supported by expert system building tools. Formalized knowledge consists of data structures, inference rules, control strategies, and languages required for implementation.

(iv) Implementation phase: During this phase, formalized knowledge is converted to a working computer program, initially called prototype of the whole system.

(v) Testing phase: This phase involves evaluating the performance and utility of prototype system and revising the system if required. The domain expert evaluated the prototype system and provides feedback, which helps the knowledge engineer to revise it.

\section{STRUCTURE OF EXPERT SYSTEM}

Expert system structure may be effectively described with the help of a diagram as shown in the figure-1, which contains important components of the system. As shown in the figure, the user interface which may use menus, natural language, or any other style of interaction. Then, an inference engine is used to reason with the expert knowledge as well the data specific to the problem being solved. Case-specific data includes both data provided by the user and partial conclusions along with certainty measures based on this data. In a simple forward chaining rule based system, case-specific data will be included in working memory. Generally all expert systems possess an explanation subsystem, which allows the program to explain its reasoning to the user. Some systems also have a knowledge acquisition module that helps the expert or knowledge engineer to easily update and check the knowledge base.

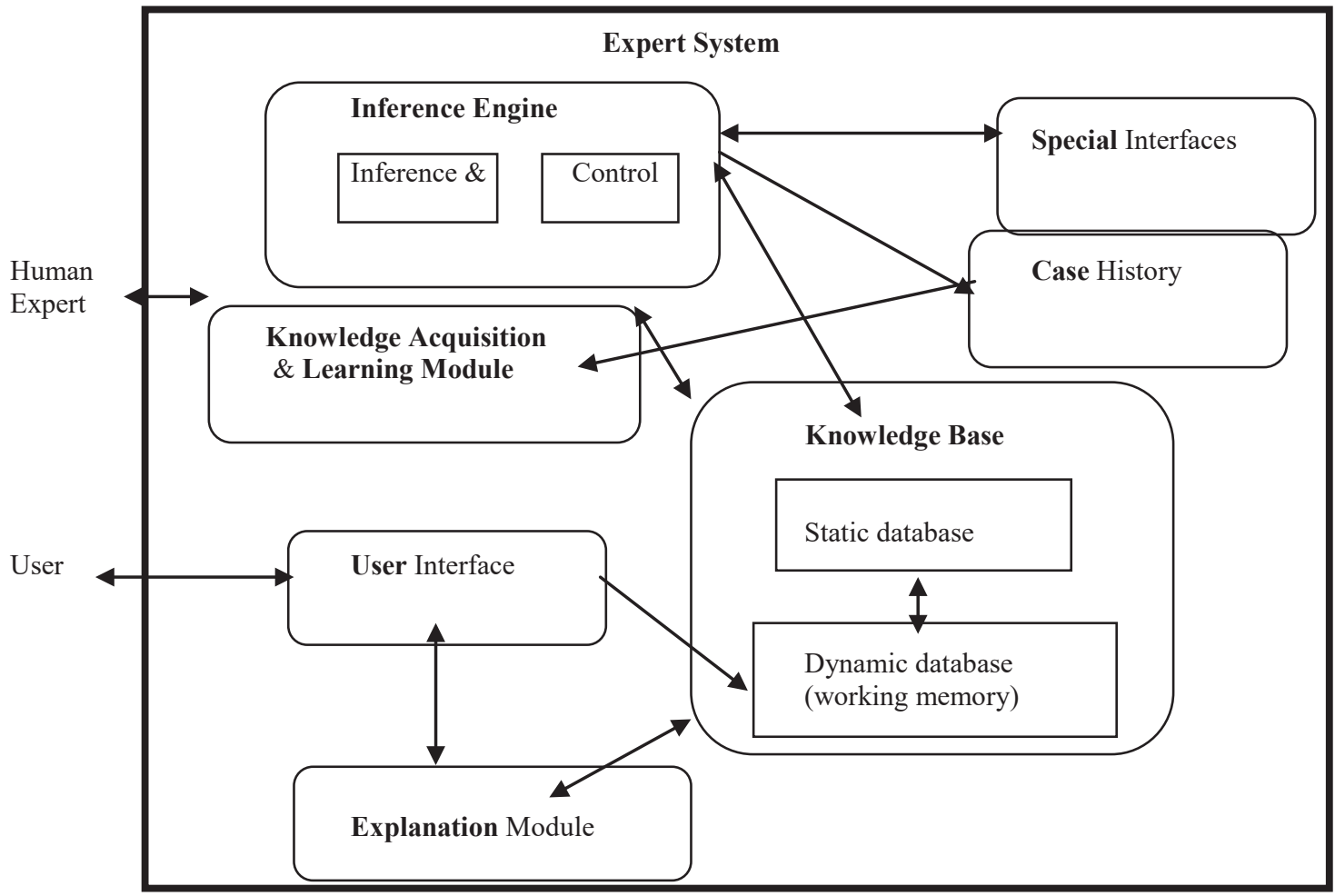

Fig 1: Structure of Expert System 
Each of these components are described as follows:

Knowledge Base:

Knowledge base of an expert system consists of knowledge regarding problem domain in the form of static and dynamic databases. Static knowledge consists of rules and facts, or any other form of knowledge representation which may be compiled as a part of the system and does not change during the execution of the system. On the other hand, dynamic knowledge consists of facts related to a particular consultation of the system collected by asking various questions to the user who is consulting the ES. At the beginning of the consultation, the dynamic knowledge base (often called working memory) is empty. Working memory is deleted at the end of consultation of the system.

\section{Inference Engine:}

An inference engine developed for an ES consists of inference mechanism as well as control strategy. The term inference refers to the process of searching through knowledge base and deriving new knowledge.

An inference rule may be defined as a statement that has two parts, an if clause and a then clause. This rule enables expert systems to find solutions to diagnostic and prescriptive problems. Inference mechanisms uses control strategy that determines the order in which rules are applied. There are mainly two types of reasoning mechanisms that use inference rules: backward chaining and forward chaining. The combination of both the reasoning processes may be used, if required.

The process of forward chaining starts with the available data and uses inference rules to conclude more data until a desired goal is achieved. An inference engine uses facts from static and dynamic knowledge bases and searches through the rules until it finds one in which the if clause is known to be true. A rule is then said to succeed. It then concludes the then clause and adds this information to the dynamic knowledge base. The inference engine continues to repeat the process until a goal is reached.

The other important inference mechanism that we are familiar with is backward chaining. Backward chaining starts with a list of goals and works backwards to see if there is data which will allow it to conclude any of these goals. An inference engine using backward chaining would search the inference rules until it finds one whose then part matches a desired goal. If the if part of the inference rule is not known to be true, then it is added to the list of goals.

\section{Knowledge Acquisition:}

A knowledge acquisition module allows the system to acquire more knowledge regarding the problem domain from experts. Interaction between knowledge engineer and the domain expert involves prolonged series of intense systematic interviews or using a questionnaire. The knowledge engineer working on a system should be able to extract expert methods, procedures, strategies, and thumb rules for solving the problem at hand. Later, the knowledge can be updated ( insertion, deletion, or updation by knowledge acquisition module of the system.

\section{Case History:}

Case History stores the file created by inference engine using the dynamic database created at the time of consultation. Useful for learning module to enrich its knowledge base. Different cases with solutions are stored in Case Base system. These cases are used for solving problem using Case Base Reasoning (CBR).

\section{User Interfaces:}

Allows user to communicate with system in interactive mode and helps system to create working knowledge for the problem to be solved.

Table 1: Dialogue Module of a User Interface

\begin{tabular}{|l|l|}
\hline \multicolumn{2}{|c|}{ Dialogue Module (User Interface) } \\
\hline System & Do you have fever? \\
\hline User & Yes \\
\hline System & Do you have bad throat? \\
\hline User & No \\
\hline System & Do you have cough? \\
\hline User & Yes \\
\hline System & Are you suffering from running nose? \\
\hline User & Yes \\
\hline System & Are you suffering from headache? \\
\hline User & No \\
\hline
\end{tabular}




\section{Explanation Module:}

Most of the expert system has explanation facilities that enables users to query the system about why it asked some questions and how it reached some conclusions; these modules are called How and Why. The sub module How tells users regarding the process through which the system has reached a particular solution, while the Why sub module explains to the user, the reasoning behind arriving at a solution to any particular problem, The questions are answered by referring to the system goals, the rules being used, and any existing problem data.

Table 2: Explanation Module

\begin{tabular}{|l|l|}
\hline User & Why module \\
\hline System & Do you have running nose? \\
\hline User & Yes. \\
\hline System & I am trying to prove that you have cold. Do you have headache? \\
\hline User & Yes \\
\hline System & Do you have sore throat? \\
\hline User & No \\
\hline System & Then you do not have cold. Do you have fever? \\
\hline User & Yes \\
\hline System & Now I am looking at the possibility of measles. Do you have cough? \\
\hline User & Yes \\
\hline System & $\begin{array}{l}\text { I can infer measles using rule "If symptoms are fever, cough, running_nose, } \\
\text { then patient has measles" measles is concluded. }\end{array}$ \\
\hline User & How Module \\
\hline System & $\begin{array}{l}\text { Since you have fever, running_nose and cough and there is a rule "If } \\
\text { symptoms are fever, cough, running_nose, then patient has measles". So } \\
\text { measles is concluded for you. }\end{array}$ \\
\hline
\end{tabular}

\section{Special Interfaces:}

It may be used for specialized activities such as handling uncertainty in knowledge. This is a major area of expert systems research that involves methods for reasoning with uncertain data and uncertain knowledge.

Knowledge is generally incomplete and uncertain. To deal with uncertain knowledge, a rule may have associated with it a confidence factor or a weight. The set of methods for using uncertain knowledge in combination with uncertain data in the reasoning process is called reasoning with uncertainty.

\section{ADVANTAGES AND DISADVANTAGES OF EXPERT SYSTEMS}

The advantages and disadvantages of ES are listed as follows:

\section{Advantages}

1. Provides improved quality of decision making

2. Domain experts are not always able to explain their logic and reasoning unlike ES.

3. Encourages organizations to clarify the logic of their decision-making.

4. Causes introduction of new products

5. Never forgets to ask a question, unlike a human.

\section{Disadvantages}

1. Unable to make creative responses as human experts would in unusual circumstances.

2. Lacks common senses needed in some decision making.

3. May cause errors in the knowledge base, and lead to wrong decisions.

4. Cannot adapt to changing environments, unless knowledge base is changed.

5. Vocabulary is often limited and difficult to understand. 


\section{APPLICATION AREAS OF EXPERT SYSTEMS}

Expert systems have been widely developed and used to solve problems in different types of domains. The appropriate problem solving technique depends generally on the type of problem and the domain.

This section will review the application of expert systems in various areas of science. Applications can be categorized into the following major classes:

Agriculture:

In the area of agriculture, expert systems have been applied to such problems as crop management, insect control, and productivity considerations for raising a given crop. Farmers and agents from the Department of Agriculture Research Services must make decisions concerning the effective and profitable production of various crops.

CROPPRO ( Baker and Lemmon 1985) was developed to aid farmers in four major areas of crop production such as: crop management problems, pest control, financial considerations, and tutoring on various crop topics

\section{Chemistry:}

The majority of expert systems were developed in the area of chemistry have been applied in a laboratory environment. The major advantage of these systems is the assistance they provide to the laboratory technician throughout a given experiment. They can assist in the planning and monitoring of the experiment, and in interpreting test data.

SPEX (Iwasaki 1982) assists scientists in planning laboratory experiments in the area of molecular biology. The scientist defines and describes the various objects to be used in an experiment. The system assists in developing a plan for achieving the goal of the experiment.

\section{Computer Science:}

Expert system applications in the computer science area have been concerned with designing or diagnosing various computer systems. Configuring computer systems which meet customer defined specifications, or diagnosing faults in a given system, can be a difficult and time consuming task.

DART ( Bennett and Hollander 1981) diagnoses faults in the hardware of computer systems. The system incorporates knowledge of the structure and expected behavior of a system in order to find design flaws in new computer systems.

\section{Engineering:}

Expert systems have been used in a wide range of applications in the area of engineering. Design, diagnostics, and control appear to dominate these applications. Expert system applications in this field can either assist an engineer in a task or replace the human operator of a control process.

PEACE ( Dincbas 1980) is an expert system developed to assist engineers in the design of electronic circuits. The system is a CAD tool which performs both synthesis and analysis of passive and digital circuits. The system uses knowledge on the functional description of the basic circuit components, coupled with topological structural constraints. The system can synthesize the circuit in defined steps which fulfills the design specification under the problem constraints.

\section{Medicine:}

The expert system can assist a physician in diagnosing medical problems of a patient or help in the interpretation of medical test results.

MYCIN is an expert system developed in 1970s at Stanford University. It was written in LISP language as the part of doctoral dissertation of Edward Shortliffe. Its job was to diagnose and recommend treatment for certain blood infections. It was developed to assist a physician who is not an expert in the field of antibiotics for the treatment of blood infections.

\section{Space Technology:}

One of the most recent applications of expert systems has been in the area of space technology. Systems are now being built for diagnosing system problems, planning mission objectives, or controlling spacecraft functions.

ECEIS ( Dickey and Toussaint 1984) controls the life support systems abroad a manned space station. After interpreting data from sensors, the system decides how to adjust the life support systems during the transition from shadow to sun. 


\section{CONCLUSION}

Expert systems have now matured and been applied to a broad range of different problems, mainly in engineering, medicine, finance, business and management. Each technology handles the uncertainty and ambiguity of human knowledge differently, and each technology has found its place in knowledge engineering. They no longer compete; rather they complement each other. A synergy of expert systems with fuzzy logic and neural computing improves adaptability, robustness, fault-tolerance and speed of knowledge-based systems. Besides, computing with words makes them more "human". It is now common practice to build intelligent systems using existing theories rather than to propose new ones, and to apply these systems to real-world problems rather than to "toy" problems. This paper has provided a brief overview of expert computer systems technology and has discussed its application in the area of science. Applications of expert systems in the sciences are expected to increase in the near future.

\section{REFERENCES}

[1] Basden A, On the Application of Expert Systems, Int. J. of Man-Machine Studies, Vol. 19, No.5, 1983, pp. 461-477

[2] Hayes-Roth F, Lenat D and Waterman D, Building Expert Systems, Addison Wesley, Reading, Mass, 1983

[3] Hayes-Roth F, Knowledge Based Systems, IEEE Computer, Vol. 17, Oct 1984, pp. 263-273

[4] Jackson P, Introduction to Expert Systems, Addison Wesley, Reading Mass, 1986

[5] Nau D S, Expert Computer Systems, IEEE Computer, Vol. 16, No. 2, Feb 1983, pp. 63-85

[6] Vadera S, Expert System Applications, Galgotia Publications, New Delhi, 1990

[7] Baker, J. and H. Lemmon 1985 Expert Systems for Agriculture, Computers and Electronics in Agriculture 1: 31-40

[8] Barr, A and E. A. Feigenbaum 1981 The Handbook of Artificial Intelligence, Vol. 1, William Kaufman Publishing

[9] 1984 The Knowledge Based Expert System: A Tutorial. IEEE Computer, Sep. pp. 11-28

[10] Mayers, W. 1986 Introduction to Expert Systems, IEEE Expert, Spr. Pp. 100-109

[11] Bruce G. Buchanan, Stanford University, Stanford, California, "Fundamentals of expert systems knowledge system laboratory

[12] D.A. Waterman, A Guide to Expert Systems, Reading, MA: Addison Wesley, 1986

[13] A.J. Gonzalez and D.D. Dankel, The Engineering of Knowledge Based Systems: Theory and Practice. Englewood Cliffs, NJ: Prentice-Hall, 1993

[14] Dutta S. 1997, Strategies For Implementing Knowledge Based Systems, 2013, IEEE Trans. Engineering Management, pp. 79-90

[15] S.J. Russel and P. Norvig, Artificial Intelligence: A Modern Approach. Englewood Cliffs, NJ: Prentice Hall, 1995

[16] Chignell M and Parsaye K, Expert Systems for Experts, John Wiley and Sons, New York, 1988

[17] Forsyth R, Expert Systems: Principles and Case Studies, Chapman and Hall, London, 1984 